\title{
A Spectral Lyapunov Function for Exponentially Stable LTV Systems
}

\author{
J. Jim Zhu, Yong Liu and Rui Hang
}

\begin{abstract}
This paper presents the formulation of a Lyapunov function for an exponentially stable linear timevarying (LTV) system using a well-defined PD-spectrum and the associated PD-eigenvectors. It provides a bridge between the first and second methods of Lyapunov for stability assessment, and will find significant applications in the analysis and control law design for LTV systems and linearizable nonlinear time-varying systems.
\end{abstract}

\section{INTRODUCTION}

Consider linear time-varying (LTV) system described by

$$
\dot{x}=A(t) x, \quad x\left(t_{0}\right)=x_{0}, \quad t \geq t_{0}
$$

where $A(t)$ is continuous and bounded for all $t \geq t_{0}$. LTV system theory is an important tool for nonlinear system control analysis and synthesis. LTV systems arise not only from time-varying parameters and structures of the system, such as the changing fuel mass and staging of a launch vehicle, changing operating environment, such as the aerodynamic coefficients of an aircraft, they also arise from linearizing a nonlinear system along a time-varying trajectory which yields LTV error dynamics.

LTV system control methods, such as linear matrix inequality control $[1,2]$ and linear parameter varying (LPV) control [3] have been developed. A differential algebraic spectra theory (DAST) for LTV systems $[4,5,6]$ extends the algebraic spectra concept of linear time-invariant (LTI) systems to LTV systems, which is a powerful tool to analyze and synthesize LTV systems. It is noted that the timevarying Parallel Differential (PD) eigenvalues defined in DAST is different from the frozen-time eigenvalues given as the roots of $\operatorname{det}[\lambda(t)-A(t)]=0$. While they are related to, they are not the same as Lyapunov, Floquet or Bohl exponents. Compared to LPV and LMI, which is essentially a linear robust control approach, DAST grasps the intrinsic nature of time-varying dynamics of LTV systems, and potentially can result in less conservative controller design and improved performance. A nonlinear control approach, called trajectory linearization control (TLC), has been developed based on DAST and successfully applied to

J. Jim Zhu is with the School of Electrical Engineering and Computer Science, Ohio University, Athens, $\mathrm{OH}, 45710$ (corresponding author, email: zhuj@ohio.edu).

Yong Liu is with Cardinal Health Inc., 22745 Savi Ranch Parkway, Yorba Linda, CA. 92887 (email: Yong.Liu.1@ohio.edu).

Rui Huang is with the School of Electrical Engineering and Computer Science, Ohio University, Athens, OH, 45710 (email: ruihuang@bobcat.ent.ohiou.edu). challenging control problems [7-12, 23, 26, 27]. One unique feature of DAST based TLC is the ability to assign timevarying closed-loop dynamics for real-time adaptation of modeling errors, changing operating conditions and objectives, and unforeseen plant degradation and faults. Adaptive time-varying bandwidth (TVB) and time-varying notch filter (TVNF) schemes based on DAST have been developed to improve the TLC stability for actuator saturation and actuator failure, such as in the direct fault tolerant control developed in [11], as well as excitation of unknown or time-dependent structural modes [12].

DAST-based stability assessment belongs to Lyapunov first method. Lyapunov second method is more widely employed in control theory and applications. However, it is difficult to apply Lyapunov second method in LTV system analysis due to the difficulty in constructing a causal and bounded Lyapunov function for stable LTV systems.

The LTV system (1) has a unique solution given an initial condition $x\left(t_{0}\right)$, which can be written as

$$
x(t)=\Phi\left(t, t_{0}\right) x\left(t_{0}\right)
$$

where $\Phi\left(t, t_{0}\right)$ is called the state transition matrix (STM). It is well known $[13,14]$ that $(1)$ is exponentially stable if and only if there exists a positive definite (p.d.) and decrescent quadratic Lyapunov function $V(t, x)$ such that

$$
0<c_{1}\|x\|_{2}^{2} \leq V(t, x)=x^{\mathrm{T}} P(t) x \leq c_{2}\|x\|_{2}^{2}, \quad \forall t
$$

where $P(t)$ is bounded, symmetric p.d., i.e.

$$
P(t)=P^{\mathrm{T}}(t), \quad 0<c_{1} I<P(t)<c_{2} I
$$

and the time-derivative of $V(t, x)$ is negative definite (n.d.) satisfying

$$
\dot{V}(t, x)=-x^{\mathrm{T}} Q(t) x \leq-c_{3}\|x\|_{2}^{2}<0
$$

where $Q(t)$ is bounded, symmetric p.d., i.e.

$$
Q(t)=Q^{\mathrm{T}}(t), \quad 0<c_{3} I<Q(t)<c_{4} I
$$

The positive constants $c_{k}$ are given by

$$
\begin{array}{ll}
c_{1}=\min _{k}\left(\inf _{t} \lambda_{k}(P(t))\right), & c_{2}=\max _{k}\left(\sup _{t} \lambda_{k}(P(t))\right) \\
c_{3}=\min _{k}\left(\inf _{t} \lambda_{k}(Q(t))\right), & c_{4}=\max _{k}\left(\sup _{t} \lambda_{k}(Q(t))\right)
\end{array}
$$

where $\lambda_{k}(X(t))$ denotes the $k$ th algebraic (frozen-time) eigenvalue of the time-varying matrix $X(t)$ as a root of the algebraic characteristic equation $\operatorname{det}[X(t)-\lambda(t) I]=0$. 
Knowing the bounds of $P(t)$ is essential in LTV system analysis and synthesis. For example, the constants $c_{k}$ can be used to estimate the envelope of the state trajectory by

$$
\|x(t)\| \leq \kappa\left\|x\left(t_{0}\right)\right\| \mathrm{e}^{-\mu\left(t-t_{0}\right)}
$$

where $\kappa=\sqrt{\frac{c_{2}}{c_{1}}}$ and $\mu=\frac{c_{3}}{2 c_{2}}$, which also asserts exponential stability by the definition. In linear adaptive control $[15,16]$ and nonlinear adaptive control $[17,18,19]$, the (constant) matrices $P$ and $Q$ for the nominal exponentially stable LTI controller are used in the adaptive control law design to assure stability of the overall adaptive system. Those results can be extended to LTV plants if practical methods can be developed for constructing the time-varying $P(t)$ and $Q(t)$ matrices.

There are two types of methods for finding time-varying $P(t)$ and $Q(t)$ matrices. The first method is by solving for a p.d. and bounded $P(t)$ from the matrix differential Lyapunov equation (DLE).

$$
\begin{aligned}
& \dot{P}(t)=-P(t) A(t)-A^{T}(t) P(t)-Q(t) \\
& P\left(t_{0}\right)=P_{0}=P_{0}^{T}, \quad t \geq t_{0}
\end{aligned}
$$

for any given p.d. and bounded $Q(t)$. It is well known that the DLE (5) has a unique bounded solution $P(t)$ if the LTV system (1) is exponentially stable. However, this method is not practical as DLE for an exponentially stable linear (LTV or LTI) system is not stable forward in time, thus in general does not provide bounded $p$. d. solutions. One approach to construct $P(t)$ is to solve the DLE using the reverse-time method by letting $\tau=-t$, rendering the DLE into an alternative form

$$
\begin{aligned}
& \dot{P}(\tau)=P(\tau) A(\tau)+A^{T}(\tau) P(\tau)+Q(\tau) \\
& P\left(\tau_{0}\right)=P_{0}=P_{0}^{T}, \quad \tau \geq \tau_{0}
\end{aligned}
$$

If the solution reaches a steady-state, that steady state can then be used as initial values for the forward integration of (5). Reverse integration requires knowledge of $A(t)$ for all future time $t \geq t_{0}$, which is an anti-causal requirement. Thus, this method is effective only when $A(t)$ has a predictable future, such as LTI system, linear periodic systems, finite-duration LTV systems with a $A(t)$ that is predefined over the operating duration, and freezes to constant at the end of the mission. In particular, for a LTI system, DLE is reduced to an Algebraic Lyapunov Equation (ALE).

$$
P A+A^{T} P+Q=0
$$

In any case, this method is not applicable for adaptive LTV systems where the closed-loop dynamics are to be synthesized in real-time in response to unforeseen changes in the plant dynamics or operating conditions. The results of the Lyapunov function for both LTI system and LTV system are summarized in [20]. In [21], the solution bounds of a general DLE (6) are analyzed. In [22], a relatively conservative solution bounds for DLE (5) is analyzed.

The second method constructs the $P(t)$ for a given $Q(t)$ using the STM by $[13,14]$

$$
P(t)=\int_{t}^{\infty} \Phi^{T}(\tau, t) Q(\tau) \Phi(\tau, t) d \tau
$$

Eq. (8) requires integration over the infinite future timeinterval, which is anti-causal, and cannot be utilized in realtime control applications. Moreover, it requires the STM $\Phi(t, \tau)$ to be known for all future time, which is not practical to obtain for LTV systems even if $A(t)$ is known for all future times, except for some special cases, such as the LTI systems.

In summary, both approaches to construct $P(t)$ for stable LTV systems are anti-causal and not applicable in real-time control application. This is not surprising as future behavior, such as exponential convergence as $t \rightarrow \infty$, of the state $x(t)$ is determined by future values of $A(t)$. The issue at hand is to find causally enforceable conditions, i.e. conditions that can be applied at the current time $t$ on $A(t)$ so that exponential stability will be guaranteed. This can be achieved using the DAST-based PD-eigenvalue assignment control $[5,6]$.

In this paper, Lyapunov function for exponentially stable LTV system is constructed using PD-eigenvalues and PD-eigenvectors, collectively called the PD-eigenstructure. Characteristics of $P(t)$ and $Q(t)$ are related to PDeigenstructure of the LTV system. Most importantly, the constructed Lyapunov function is causal and applicable to real-time applications.

The developed Lyapunov function is a useful tool not only as an alternative to the ALE (7) for solving $P$ and $Q$ in many Lyapunov control methods for time-invariant linear and nonlinear systems, such as adaptive control, but also for extending those methods to LTV and linearizable nonlinear time varying (NLTV) systems. Using the results developed in this paper, it is possible to assign the nominal controller as an LTV system, the causal $P(t)$ and $Q(t)$ matrix can be used in the adaptive law, as illustrated in [23]. Thus there is potential to design a less conservative and more robust adaptive control system. The main results of this paper also establish an alternative proof for the PD-eigenvalue stability criterion, along with estimate of the exponential envelope of the state trajectory.

Following this introductory section, Section II presents a brief overview of the DAST to facilitate exposition of the main results, which are presented in Section III. Some illustrative examples are presented in Section IV. The paper is concluded with Section V.

\section{OVERVIEW OF THE DAST}

In this section we provide a brief overview of the DAST $[4,5,6]$. Consider the $n$-dimensional NLTV tracking error or operating error dynamics

$$
\dot{x}=f(t, x), \quad x\left(t_{0}\right)=x_{0}, \quad t \geq t_{0}
$$

where $f$ is continuous in $t$ and differentiable with respect to $x$, and $x(t) \equiv 0$ is the desired equilibrium state that needs to be stabilized. The time-dependence of $f$ may be due to either time-varying parameters or time-varying nominal 
tracking trajectory of the NLTV system. To begin with, we consider the special case where (9) can be linearized at the null equilibrium point (i.e. along the nominal trajectory) to a LTV system of the form

$$
\dot{x}=A_{\mathrm{c}}(t) x, \quad x\left(t_{0}\right)=x_{0}, \quad t \geq t_{0}
$$

where $A_{\mathrm{c}}(t)$ is continuous and bounded, and is in the companion canonical form

$$
A_{\mathrm{c}}(t)=\left[\begin{array}{c|ccc}
0 & & & \\
\vdots & & I_{n-1} & \\
0 & & & \\
\hline-\alpha_{1}(t) & -\alpha_{2}(t) & \cdots & -\alpha_{n}(t)
\end{array}\right]
$$

where $I_{n-1}$ is the $(n-1) \times(n-1)$ identity matrix. By letting $y=x_{1}$, the state equation (10) is equivalent to an $n$ th-order scalar LTV differential equation

$$
\begin{gathered}
y^{(n)}+\alpha_{n}(t) y^{(n-1)}+\cdots+\alpha_{2}(t) \dot{y}+\alpha_{1}(t) y=0 \\
y^{(k-1)}\left(t_{0}\right)=x_{0_{k}}, k=1, \ldots n \quad t \geq t_{0}
\end{gathered}
$$

The general solution to LTV equation (11) can be written as

$$
y(t)=\sum_{k=1}^{n} C_{k} y_{k}(t)=\sum_{k=1}^{n} C_{k} \mathrm{e}^{\int_{t_{0}}^{t} \rho_{k}(\tau) d \tau}
$$

where $\left\{y_{k}(t)=\exp \left(\int_{t_{0}}^{t} \rho_{k}(\tau) \mathbf{d} \tau\right)\right\}_{k=1}^{n}$ is a set of linearly independent solutions to (11), and $\left\{\rho_{k}(t)\right\}_{k=1}^{n}$ is called a parallel differential (PD) spectrum for (11) in which each $\rho_{k}(t)$ is called a $P D$-eigenvalue for $A_{\mathrm{c}}(t)$. The PDeigenvalues are solutions to an $(n-1)$ th-order NLTV differential equation, called $P D$-characteristic equation

$$
\begin{aligned}
\Delta(\rho)= & \mathcal{D}_{\rho}^{n-1}(\rho)+\alpha_{n}(t) \mathcal{D}_{\rho}^{n-2}(\rho)+ \\
& \cdots+\alpha_{3}(t) \mathcal{D}_{\rho}(\rho)+\alpha_{2}(t) \rho+\alpha_{1}(t)=0
\end{aligned}
$$

where $\quad \mathcal{D}_{\rho}(\rho)=\left[\frac{d}{d t}+\rho(t)\right](\rho(t))=\dot{\rho}(t)+\rho^{2}(t), \quad$ and $\mathcal{D}_{\rho}^{k}=\mathcal{D}_{\rho} \mathcal{D}_{\rho}^{k-1}$. In particular, for $n=2$, the PD-character equation is given by the Ricatti equation

$$
\dot{\rho}+\rho^{2}+\alpha_{2}(t) \rho+\alpha_{1}(t)=0
$$

Note that for real-valued coefficients $\alpha_{k}(t)$, the PDeigenvalues $\rho_{k}(t)$ may be complex-valued in order to avoid singular solutions. Moreover, the synthesis formula for $\alpha_{k}(t)$ from a PD-spectrum $\left\{\rho_{k}(t)\right\}_{k=1}^{n}$ is given by

$$
\begin{aligned}
& \alpha_{1}(t)=\rho_{1}(t) \rho_{2}(t)+\frac{\rho_{1}(t) \dot{\rho}_{2}(t)-\rho_{2}(t) \dot{\rho}_{1}(t)}{\rho_{2}(t)-\rho_{1}(t)} \\
& \alpha_{2}(t)=-\rho_{1}(t)-\rho_{2}(t)-\frac{\dot{\rho}_{2}(t)-\dot{\rho}_{1}(t)}{\rho_{2}(t)-\rho_{1}(t)}
\end{aligned}
$$

For each PD-eigenvalue $\rho_{i}(t)$, an associated PDeigenvector $v_{i}(t)$ for $A_{c}(t)$ is defined by

$$
A_{c}(t) \mu_{i}(t)-\rho_{i}(t) \mu_{i}(t)=\dot{\mu}_{i}(t)
$$

where $\mu_{i}(t)$ can be expressed using the PD-eigenvalue $\rho_{i}(t)$ as $\mu_{i}(t)=\left[\begin{array}{lllll}1 & \mathcal{D}_{\rho_{i}}\{1\} & \mathcal{D}_{\rho_{i}}^{2}\{1\} & \cdots & \mathcal{D}_{\rho_{i}}^{n-1}\{1\}\end{array}\right]^{\mathrm{T}}$. The diagonal matrix

$$
\Upsilon(t)=\operatorname{diag}\left[\rho_{1}(t), \rho_{2}(t), \cdots, \rho_{n}(t)\right]
$$

is called a PD-spectral canonical form for $A_{c}(t)$, and the matrix consisting of all corresponding PD-eigenvectors

$$
\begin{aligned}
M(t) & =\left[\mu_{1}(t)\left|\mu_{2}(t)\right| \cdots \mid \mu_{n}(t)\right] \\
& =\left[\begin{array}{cccc}
1 & 1 & \cdots & 1 \\
\mathcal{D}_{\rho_{1}}(1) & \mathcal{D}_{\rho_{2}}(1) & \cdots & \mathcal{D}_{\rho_{n}}(1) \\
\mathcal{D}_{\rho_{1}}^{2}(1) & \mathcal{D}_{\rho_{2}}^{2}(1) & \cdots & \mathcal{D}_{\rho_{n}}^{2}(1) \\
\vdots & & \ddots & \vdots \\
\mathcal{D}_{\rho_{1}}^{n-1}(1) & \cdots & \cdots & \mathcal{D}_{\rho_{n}}^{n-1}(1)
\end{array}\right]
\end{aligned}
$$

is called a PD-modal matrix for $A_{c}(t)$, and for the LTV system (10). A PD-spectrum (PD-spectral matrix) is said to be well-defined if the PD-eigenvalues $\rho_{k}(t)$ are bounded and continuously differentiable at least $n$ times. A PD-modal matrix is said to be bounded diffeomorphic if it is continuously differentiable and invertible, with a uniform bound on $M(t), M^{-1}(t)$, and $\dot{M}(t)$. A bounded diffeomorphic PD-modal matrix constitutes a (stability preserving) Lyapunov transformation that relates the PD-spectral matrix $\Upsilon(t)$ and $A_{c}(t)$ by

$$
\Upsilon(t)=M^{-1}(t)\left[A_{c}(t) M(t)-\dot{M}(t)\right]
$$

The above concepts can be extended to general LTV system (1) via a Lyapunov transformation that reduces $A(t)$ to $A_{c}(t)$

$$
A_{c}(t)=L^{-1}(t)[A(t) L(t)-\dot{L}(t)]
$$

Then the PD-spectral matrix $\Upsilon(t)$ for $A_{c}(t)$ is called a PDspectral matrix for $A(t)$, and the composite Lyapunov transformation $V(t)=L(t) M(t)$ is called the corresponding modal matrix for $A(t)$. A sufficiency condition for the existence of such a bounded diffeomorphism $L(t)$ is that there exists a bounded, sufficiently differentiable $n \times 1$ matrix $B(t)$ such that $\{A(t), B(t)\}$ is strongly controllable, i.e. $\inf \left|\operatorname{det}\left[B(t)\left|\mathcal{P}_{A} B(t)\right| \cdots \mid \mathcal{P}_{A}{ }^{n-1} B(t)\right]\right|=c>0$, where $\mathcal{P}_{A} B(t)=\dot{B}(t)-A(t) B(t), \mathcal{P}_{A}^{k}=\mathcal{P}_{A} \mathcal{P}_{A}^{k-1}$

The PD-eigenvalue concept is a natural extension of the eigenvalue concept for LTI systems in that, when the coefficients $\alpha_{k}(t) \equiv \alpha_{k}=$ constant, the PD-character (differential) equation (13) possesses constant solutions $\rho_{k}(t) \equiv \rho_{k}=$ constant that are conventional characteristic roots for (11), and eigenvalues for $A_{\mathrm{c}}(t) \equiv A_{\mathrm{c}}$, for which (13) reduces to the conventional algebraic characteristic equation, and the synthesis formula (15) reduces to the conventional polynomial coefficient synthesis formula. Moreover, stability of the LTV system (10) or (11), and consequently the null equilibrium of the NLTV system (9), can be determined by the negativity of the real-part of the PD-eigenvalues. In particular, exponential stability is implied by $\operatorname{Re}\left(\rho_{k}(t)\right)<-c<0$, for some constant $c>0$, and for all $k$. 
It is noted that, since $\left\{y_{k}(t)\right\}_{k=1}^{n}$ are linearly independent, by definition PD-eigenvalues are always distinct. For instance, given a second order LTI system

$$
\dot{x}=\left[\begin{array}{cc}
0 & 1 \\
-\lambda^{2} & -2 \lambda
\end{array}\right] x
$$

the system has repeated eigevalue $\lambda$, whereas a PD-spectrum can be chosen as $\rho_{1}(t) \equiv \lambda$ and $\rho_{2}(t)=\lambda+\frac{1}{t+d}$ for some constant $d \leq t_{0}$. It should be noted that PD-eigenvalues are functions of time. They are different from Lyapunov exponent, Floquet exponent or Bohl exponent, which have constant values. PD-eigenvalues are also different from the frozen-time eigenvalues of an LTV system. It is well known that frozen-time eigenvalues given as the roots of $\operatorname{det}[\lambda(t)-A(t)]=0$ are in general neither sufficient nor necessary for stability assessment of a LTV system. Since evaluating a PD-spectrum for an arbitrary given matrix $A(t)$ entails solving the $(n-1)$ th-order NLTV PD-characteristic (differential) equation, which in general is difficult. Thus at the present time using PD-spectrum as an analysis tool remains a challenge, and requires significant future research. However, PD-spectrum as a design synthesis tool is not only practical, and very effective, as has been demonstrated in $[7-12,23,26,27]$. The main results of this paper that follow bridge an important gap between the Lyapunov First Method based PD-spectral theory and the Lyapunov Second Method based control design techniques.

\section{MAIN RESULTS}

The main result of this paper establishes a relationship between the first and second method of Lyapunov for stability assessment using the PD-spectral concept.

Theorem 1. Let $\Upsilon(t)=\operatorname{diag}\left[\rho_{1}(t), \rho_{2}(t), \cdots, \rho_{n}(t)\right]$ be a well defined PD-spectral matrix for $A(t)$ with a bounded diffeomorphic PD-modal matrix $M(t)=\left[\mu_{1}(t)\left|\mu_{2}(t)\right| \cdots \mid\right.$ $\left.\mu_{n}(t)\right]$, where $\mu_{k}(t)$ are the corresponding $P D$-eigenvectors for the PD-eigenvalues $\rho_{k}(t)$. If

$$
\operatorname{Re}\left(\rho_{k}(t)\right)<-c<0
$$

for some positive constants $c$, then the null equilibrium for $\dot{x}=A(t) x$ is exponentially stable. Moreover, $V_{x}(t, x)=$ $x^{*}(t) P_{x}(t) x(t)$ is a Lyapunov function for $\dot{x}=A(t) x$ with $\dot{V}_{x}(t, x)=-x^{*}(t) Q_{x}(t) x(t)$, where

$$
P_{x}(t)=M^{*-1}(t) M^{-1}(t)
$$

and

$$
Q_{x}(t)=-2 M^{*-1}(t) \operatorname{Re}(r(t)) M^{-1}(t)
$$

where * denotes the conjugate transpose.

Proof of Theorem 1 relies on the following lemma, which is an important result in its own right.

Lemma 1. Let

$$
P_{z}(t)=\int_{t}^{\infty} \Phi_{z}^{*}(\tau, t) Q_{z}(\tau) \Phi_{z}(\tau, t) d \tau
$$

where $Q_{z}(t)$ is a continuous, bounded, positive definite, symmetric matrix, and $\Phi_{z}(t, \tau)$ is the state transition matrix for the LTV system $\dot{z}=A_{z}(t) z$. Let $M(t)$ be a Lyapunov coordinate transformation and define

$$
x(t)=M(t) z(t)
$$

If the null equilibrium point for the LTV system $\dot{z}=A_{z}(t) z$ is exponentially stable, then $V_{x}(t, x)=x^{*}(t) P_{x}(t) x(t)$ is a Lyapunov function for $\dot{x}=A_{x}(t) x$ with $\dot{V}_{x}(t, x)=$ $-x^{*}(t) Q_{x}(t) x(t)$, where

$$
\begin{gathered}
A_{x}(t)=M^{-1}(t) A_{z}(t) M(t)-M^{-1}(t) \dot{M}(t) \\
P_{x}(t)=M^{*-1}(t) P_{z}(t) M^{-1}(t) \\
Q_{x}(t)=M^{*-1}(t) Q_{z}(t) M^{-1}(t)
\end{gathered}
$$

Proof. Suppose the null equilibrium point for the LTV system $\dot{z}=A_{z}(t) z$ is exponentially stable and let

$$
P_{z}(t)=\int_{t}^{\infty} \Phi_{z}^{*}(\tau, t) Q_{z}(\tau) \Phi_{z}(\tau, t) d \tau
$$

It can be verified that $V_{z}(t, z)=z^{*}(t) P_{z}(t) z(t)$ is a Lyapunov function for $\dot{z}=A_{z}(t) z$ with $\dot{V}_{z}(t, z)=$ $-z^{*}(t) Q_{z}(t) z(t)$ [13, pp. 155-159]. Let $M(t)$ be a Lyapunov coordinate transformation and define

$$
x(t)=M(t) z(t)
$$

Then the null equilibrium for the LTV system $\dot{x}=A_{x}(t) x$ is also exponentially stable, where

$$
A_{x}(t)=M^{-1}(t) A_{z}(t) M(t)-M^{-1}(t) \dot{M}(t)
$$

Let

and

$$
P_{x}(t)=M^{*-1}(t) P_{z}(t) M^{-1}(t)
$$

Note that

$$
Q_{x}(t)=M^{*-1}(t) Q_{z}(t) M^{-1}(t)
$$

$$
\begin{aligned}
z(t) & =M^{-1}(t) x(t) \\
\Phi_{x}(\tau, t) & =M(\tau) \Phi_{z}(\tau, t) M^{-1}(t) \\
\Phi_{x}^{*}(\tau, t) & =M^{*-1}(t) \Phi_{z}^{*}(\tau, t) M^{*}(\tau)
\end{aligned}
$$

Then

$$
\begin{aligned}
& P_{x}(t)=M^{*-1}(t) P_{z}(t) M^{-1}(t) \\
& =M^{*-1}(t) \int_{t}^{\infty} \Phi_{z}^{*}(\tau, t) Q_{z}(\tau) \Phi_{z}(\tau, t) d \tau M^{-1}(t) \\
& =\int_{t}^{\infty} M^{*-1}(t) \Phi_{z}^{*}(\tau, t) M^{*}(\tau) M^{*-1}(\tau) Q_{z}(\tau) M^{-1}(\tau) M(\tau) \Phi_{z}(\tau, t) M^{-1}(t) d \tau \\
& =\int_{t}^{\infty} \Phi_{x}^{*}(\tau, t) Q_{x}(\tau) \Phi_{x}(\tau, t) d \tau
\end{aligned}
$$

It follows from [13, pp. 155-159] that $V_{x}(t, x)=$ $x^{*}(t) P_{x}(t) x(t)$ is a Lyapunov function for $\dot{x}=A_{x}(t) x$ with $\dot{V}_{x}(t, x)=-x^{*}(t) Q_{x}(t) x(t)$.

Proof of Theorem 1. Suppose the PD-spectral matrix $r(t)$ satisfies (17) and the PD-modal matrix $M(t)$ is bounded and diffeomorphic. Let $z(t)=M^{-1}(t) x(t)$. Then $\dot{z}=\Upsilon(t) z$. 
Let $Q_{z}(t)=-2 \operatorname{Re}(Y(t))>2 c I>0$. It is readily verified that $P_{z}=I$ satisfies the Lyapunov equation

$$
\dot{P}_{z}=-P_{z} \Upsilon(t)-\Upsilon^{*}(t) P_{z}^{*}-Q_{z}(t)
$$

Thus, $V_{z}(t, z)=z^{*}(t) P_{z}(t) z(t)=z^{*}(t) z(t)$ is a Lyapunov function for $\dot{z}=\Upsilon(t) z$ with $\dot{V}_{z}(t, z)=2 z^{*}(t) \operatorname{Re}(\Upsilon(t)) z(t)$ $<0$. By Theorems 4.10 and 4.12 of [13], the null equilibrium of $\dot{z}=\Upsilon(t) z$ is exponentially stable. Since $M(t)$ constitutes a Lyapunov transformation, the null equilibrium for $\dot{x}=A(t) x$ is also exponentially stable. It then follows from Lemma 1 that $V_{x}(t, x)=x^{*}(t) P_{x}(t) x(t)$ is a Lyapunov function for $\dot{x}=A(t) x$ with $\dot{V}_{x}(t, x)=$ $-x^{*}(t) Q_{x}(t) x(t)$, where $P_{x}(t)$ and $Q_{x}(t)$ are given by (18) and (19), respectively.

Remark 1. The proof of Theorem 1 provides an insight into the relationship between Lyapunov first method and second method for LTV systems. In particular, in the PD-spectral coordinates $\dot{z}=\Upsilon(t) z$, where the modes of the LTV systems are decoupled, the Lyapunov function $V_{z}(t, z)=$ $z^{*}(t) P_{z}(t) z(t)=z^{*}(t) z(t)$ represents the total energy in the modes, and $\dot{V}_{z}(t, z)=2 z^{*}(t) \operatorname{Re}(r(t)) z(t)$ gives the energy decay rate in terms of the PD-spectrum, which is twice the exponential decay rate of the envelope of the trajectories. The energy level and decay rate are "scaled" by the condition number of the PD-modal matrix in the original coordinates for the LTV system $\dot{x}=A(t) x$.

Remark 2. For LTI systems with distinct eigenvalues, the conventional (algebraic) eigenvalues and eigenvectors define a constant PD-spectrum and a constant PD-modal matrix. The bounds on the Lyapunov function $V(x)=x^{*}(t) P x(t)$ and on the derivative $\dot{V}(x)=-x^{*}(t) Q x(t)$ given by (3) can be used to estimate the envelope of the state trajectory by (4). Notice that, by letting $P=M^{*-1} M^{-1}$, the eigenvalues $\lambda_{k}(P)=\sigma_{k}^{2}\left(M^{-1}\right)$, which are the singular value squared for $M^{-1}$. Thus, the estimate of the peaking bound $\kappa=\sqrt{c_{2} / c_{1}}$ is actually the condition number of the (PD) modal matrix $M$, which is an indication of how singular the modal matrix is. In particular, the condition number attains its minimum when the modal matrix is unitary, in which case the peaking bound is $\kappa=1$. Thus, the peaking phenomenon is determined by the orthoganality of the PD-modal matrix, rather than by the exponential decay rate. Moreover, when $M$ is unitary, $M^{-1}=M^{*}$, then $P=M^{*-1} M^{-1}=I$, and $\lambda_{k}(Q)=$ $-2 \lambda_{k}(\operatorname{Re}(r))$. Thus, the exponential decay rate $\mu=-c_{3} /\left(2 c_{2}\right)=-\min _{k} \mid \operatorname{Re}\left(\lambda_{k}(\Upsilon) \mid\right.$, which is decay rate of the slowest mode. It follows that when two eigenvectors are nearly collinear, not only peaking of the trajectory will be large, the decay rate will also be slower than the single slowest mode. In practice, if there are design freedoms to assign the eigenvectors, they should be assigned as orthogonal as possible. These observations also apply to LTV case with a suitably defined induced matrix norm, such as $\|A(\cdot)\|_{2}=\sup _{t \geq t_{0}}\|A(t)\|_{2}$.
Remark 3. The technical difficulty for obtaining a causal expression of a Lyapunov function for an exponentially stable LTV system is circumvented by requiring the realpart of PD-spectrum to be bounded by a negative number. This result allows time-varying closed-loop dynamics to be synthesized for adaptation to unforeseen modeling errors and adverse operating conditions as they arise, thereby achieving best tradeoff between the closed-loop system performance, robustness and control energy consumption.

Remark 4. For LTI systems, Theorem 1 provides an alternative method to the ALE for computing a Lyapunov function. Different $P$ matrix can be obtained using different modal matrices, which amounts to choosing a different coordinate for the state space.

Remark 5. Theorem 1 also provides an alternative proof for the PD-eigenvalue stability criterion.

\section{ILLUSTRATIVE EXAMPLES}

In this section we use second-order LTI and LTV system examples to illustrate the utility of the main results, and to gain some insight into the relationship between the first and second methods of Lyapunov. We will also demonstrate the fallacy of frozen-time eigenvalue concept for LTV systems.

\subsection{LTI Examples}

In this subsection we consider some LTI examples with distinct eigenvalues, which will be denoted $\lambda_{k}$.

Example 1 (The Companion Form, Distinct Real Eigenvalues). Suppose $A$ is in the companion canonical form

$$
A=\left[\begin{array}{cc}
0 & 1 \\
-a_{1} & -a_{2}
\end{array}\right]=\left[\begin{array}{cc}
0 & 1 \\
-\lambda_{1} \lambda_{2} & \lambda_{1}+\lambda_{2}
\end{array}\right]
$$

Then the modal matrix is given by

$$
M=\left[\begin{array}{cc}
1 & 1 \\
\lambda_{1} & \lambda_{2}
\end{array}\right]
$$

The $P, Q$ matrices are given in terms of the eigenvalues by

$$
\begin{gathered}
P=\left[\begin{array}{cc}
\frac{\lambda_{1}^{2}+\lambda_{2}^{2}}{\left(\lambda_{2}-\lambda_{1}\right)^{2}} & \frac{-\left(\lambda_{1}+\lambda_{2}\right)}{\left(\lambda_{2}-\lambda_{1}\right)^{2}} \\
\frac{-\left(\lambda_{1}+\lambda_{2}\right)}{\left(\lambda_{2}-\lambda_{1}\right)^{2}} & \frac{2}{\left(\lambda_{2}-\lambda_{1}\right)^{2}}
\end{array}\right] \\
Q=\left[\begin{array}{cc}
\frac{-2 \lambda_{1} \lambda_{2}\left(\lambda_{1}+\lambda_{2}\right)}{\left(\lambda_{2}-\lambda_{1}\right)^{2}} & \frac{4 \lambda_{1} \lambda_{2}}{\left(\lambda_{2}-\lambda_{1}\right)^{2}} \\
\frac{4 \lambda_{1} \lambda_{2}}{\left(\lambda_{2}-\lambda_{1}\right)^{2}} & \frac{-2\left(\lambda_{1}+\lambda_{2}\right)}{\left(\lambda_{2}-\lambda_{1}\right)^{2}}
\end{array}\right]
\end{gathered}
$$

Note that in this case, $M$ cannot be orthogonal when both eigenvalues are of the same sign. Thus, peaking is unavoidable. For $a_{1}=2, a_{2}=3$, we have $\lambda_{1}=-1$, $\lambda_{2}=-2$, and

$$
P=\left[\begin{array}{ll}
5 & 3 \\
3 & 2
\end{array}\right], \quad Q=\left[\begin{array}{cc}
12 & 8 \\
8 & 6
\end{array}\right]
$$

with the singular values $\sigma\left(M^{-1}\right)=\{2.6180,0.3820\}$, condition number $\kappa(M)=6.8541$, and the eigenvalues $\lambda(Q)=\{17.5440,0.4560\}$. Thus the estimated trajectory 
envelope is

$$
\|x(t)\| \leq 6.8541\left\|x\left(t_{0}\right)\right\| \mathrm{e}^{-0.0333\left(t-t_{0}\right)}
$$

which should be compared to the slowest mode $\mathrm{e}^{-\left(t-t_{0}\right)}$.

Example 2 (Orthonormal Modal Matrix, Distinct Real Eigenvalues). Consider

$$
A=\left[\begin{array}{cc}
-1.5 & 0.5 \\
0.5 & -1.5
\end{array}\right]
$$

with eigenvalues $\lambda_{1}=-1, \lambda_{2}=-2$. The modal matrix is given by

$$
M=\left[\begin{array}{cc}
0.7071 & 0.7071 \\
-0.7071 & 0.7071
\end{array}\right]
$$

Note that $M$ is orthonormal, and

$$
P=\left[\begin{array}{ll}
1 & 0 \\
0 & 1
\end{array}\right], \quad Q=\left[\begin{array}{cc}
12 & 8 \\
8 & 6
\end{array}\right]
$$

with $\sigma\left(M^{-1}\right)=\{1,1\}, \kappa(M)=1$, and $\lambda(Q)=\{4,2\}$. Thus the estimated trajectory envelope is

$$
\|x(t)\| \leq\left\|x\left(t_{0}\right)\right\| \mathrm{e}^{-\left(t-t_{0}\right)}
$$

which should be compared to Example 1 above.

Example 3 (The Companion Form, Complex Eigenvalues). Suppose $A$ is in the companion canonical form

$$
A=\left[\begin{array}{cc}
0 & 1 \\
-a_{1} & -a_{2}
\end{array}\right]=\left[\begin{array}{cc}
0 & 1 \\
-\left(\sigma^{2}+\omega^{2}\right) & 2 \sigma
\end{array}\right]
$$

Then the modal matrix is given by

$$
M=\left[\begin{array}{cc}
1 & 1 \\
\sigma+j \omega & \sigma-j \omega
\end{array}\right]
$$

The $P, Q$ matrices are given in terms of the eigenvalues by

$$
P=\left[\begin{array}{cc}
\frac{\left(\sigma^{2}+\omega^{2}\right)}{22 \omega^{2}} & \frac{-\sigma}{2 \omega^{2}} \\
\frac{-\sigma}{2 \omega^{2}} & \frac{1}{2 \omega^{2}}
\end{array}\right], Q=\left[\begin{array}{cc}
\frac{-\sigma\left(\sigma^{2}+\omega^{2}\right)}{\omega^{2}} & \frac{\sigma^{2}}{\omega^{2}} \\
\frac{\sigma^{2}}{\omega^{2}} & \frac{-\sigma}{\omega^{2}}
\end{array}\right]=-2 \sigma P
$$

Note that in this case, $M$ cannot be unitary. Thus, peaking is unavoidable. For $a_{1}=1, \quad a_{2}=\sqrt{2}$, we have $\lambda_{1,2}=-0.7071 \pm j 0.7071$, and

$$
P=\left[\begin{array}{cc}
2 & 1.4142 \\
1.4142 & 2
\end{array}\right], \quad Q=\left[\begin{array}{cc}
2.8284 & 2 \\
2 & 2.8284
\end{array}\right]
$$

with $\sigma\left(M^{-1}\right)=\{0.7654,1.8478\}, \kappa(M)=2.4142$ and $\lambda(Q)=\{0.8284,4.8284\}$. Thus the estimated trajectory envelope is

$$
\|x(t)\| \leq 2.4142\left\|x\left(t_{0}\right)\right\| \mathrm{e}^{-0.1213\left(t-t_{0}\right)}
$$

which should be compared to the envelope defined by the real-part of the eigenvalues $\mathrm{e}^{-0.7071\left(t-t_{0}\right)}$.

Example 4 (Unitary Modal Matrix, Complex Eigenvalues). Consider

$$
A=\left[\begin{array}{cc}
-0.7071 & 0.7071 \\
-0.7071 & -0.7071
\end{array}\right]
$$

with eigenvalues $\lambda_{1,2}=-0.7071 \pm j 0.7071$. The modal matrix is given by

$$
M=\left[\begin{array}{cc}
0.7071 & 0.7071 \\
0.7071 j & -0.7071 j
\end{array}\right]
$$

which is unitary, and

$$
P=\left[\begin{array}{ll}
1 & 0 \\
0 & 1
\end{array}\right], \quad Q=\left[\begin{array}{cc}
1.414 & 0 \\
0 & 1.414
\end{array}\right]
$$

with $\quad \sigma\left(M^{-1}\right)=\{1,1\}, \quad \kappa(M)=1, \quad$ and $\quad \lambda(Q)=$ $\{1.414,1.414\}$. Thus the estimated trajectory envelope is

$$
\|x(t)\| \leq\left\|x\left(t_{0}\right)\right\| \mathrm{e}^{-7071\left(t-t_{0}\right)}
$$

which should be compared to Example 3 above.

\subsection{LTV Examples}

In this subsection we first consider a LTV example that was developed to demonstrate the fallacy of frozen-time eigenvalue concept for LTV systems defined by the roots of $\operatorname{det}[A(t)-\lambda(t) I]=0$. We will compare the Lyapunov function derived from the PD-eigenvalue concept and the fallacious frozen-time eigenvalue concept. A third LTV example is given to illustrate application of the main result to a time-varying bandwidth (TVB) filter.

Example 5 (Parameterized Marcus-Yamabe Equation). Consider the parameterized Marcus-Yamabe (M-Y) Equation (1) with [24]

$$
A(t)=\left[\begin{array}{cc}
a-\frac{1}{2} \omega+a \cos (\omega t) & \frac{1}{2} \omega-a \sin (\omega t) \\
-\frac{1}{2} \omega-a \sin (\omega t) & a-\frac{1}{2} \omega-a \cos (\omega t)
\end{array}\right]
$$

which was developed to show the failure of the sufficiency of the stability assessment using the negative real-part criterion using the frozen-time eigenvalue. The PD-spectral and PD-modal matrices are given by $\Upsilon(t)=\operatorname{diag}\left[2 a-\frac{1}{2} \omega,-\frac{1}{2} \omega\right]$ and

$$
M(t)=\left[\begin{array}{cc}
\cos \left(\frac{1}{2} \omega t\right) & \sin \left(\frac{1}{2} \omega t\right) \\
-\sin \left(\frac{1}{2} \omega t\right) & \cos \left(\frac{1}{2} \omega t\right)
\end{array}\right]
$$

Note that the PD-eigenvalues are constant and real, and exponential stability will be achieved for $\omega>4 a>0$. Note also that the PD-modal matrix is orthonormal for all $t$. The $P(t)$ and $Q(t)$ matrices are given in terms of the parameters $a, \omega$ by $P(t)=I$ and

$$
Q(t)=\left[\begin{array}{cc}
-2 a+\omega-2 a \cos (\omega t) & -2 a \sin (\omega t) \\
-2 a \sin (\omega t) & -2 a+\omega+2 a \cos (\omega t)
\end{array}\right]
$$

The eigenvalues for $Q(t)$ are given by $\{\omega,-4 a+\omega\}$. Thus $Q(t)>0$ for $\omega>4 a$, in agreement with the PD-spectral stability condition. The parameterized M-Y equation specialized to the original M-Y equation [13, Example 4.22, p.158] when $\omega=2$ and $a=0.75$, for which the PD- 
eigenvalues are $\rho_{1}(t)=0.5, \rho_{2}(t)=-1$. By the PD-spectral stability criterion, the system is unstable. This is also verified with the fact that for these values $Q(t)<0$ for all $t$, and $\dot{V}(t, x)=-x^{*}(t) Q(t) x(t)>0$, for all $t$. Thus, any initial value $x\left(t_{0}\right)$ will move away from the null equilibrium $x=0$. Now, consider the frozen-time eigenvalues for the original M-Y equation, which are $\lambda_{1,2}(t)=-\frac{1}{4} \pm j \frac{\sqrt{7}}{4}$, which by the fallacious frozen-time eigenvalue stability criterion would imply exponential stability. The frozen-time modal matrix can be easily found to be

$$
M_{\mathrm{FT}}(t)=\left[\begin{array}{cc}
1-\frac{3}{4} \sin (2 t) & 1-\frac{3}{4} \sin (2 t) \\
-\frac{3}{4} \cos (2 t)+i \frac{\sqrt{7}}{4} & -\frac{3}{4} \cos (2 t)-i \frac{\sqrt{7}}{4}
\end{array}\right]
$$

This would lead to a positive definite $Q(t)>0$ with eigenvalues $\quad \lambda(Q(t))=\left\{\frac{4}{4-3 \sin (2 t)}, \frac{4}{7[4-3 \sin (2 t)]}\right\}, \quad$ which would also (fallaciously) imply exponential stability. However, with the correct time-varying coordinate transformation and with the frozen-time eigenvalues treated as PD-eigenvalues, this stable system would actually be given by $\dot{x}=B(t) x$, where

$$
\begin{aligned}
B(t) & =\left(M_{\mathrm{FT}}(t) \operatorname{diag}\left[\lambda_{1}(t), \lambda_{2}(t)\right]+\dot{M}_{\mathrm{FT}}(t)\right) M_{\mathrm{FT}}^{-1}(t) \\
& =\left[\begin{array}{cc}
\frac{8-6 \sin (2 t)+9 \sin (4 t)+24 \cos (2 t)}{24 \sin (2 t)-32} & \frac{3}{4} \sin (2 t)+1 \\
\frac{3}{4} \sin (2 t)-\frac{1}{4} & \frac{23-48 \sin (2 t)+9 \cos (4 t)}{24 \sin (2 t)-32}
\end{array}\right] *
\end{aligned}
$$

Example 6 (A time-varying bandwidth filter). Consider an LTV system (1) with

$$
A(t)=\left[\begin{array}{cc}
0 & 1 \\
-\omega_{n}^{2}(t) & -2 \zeta \omega_{n}(t)-\frac{\dot{\omega}_{n}(t)}{\omega_{n}(t)}
\end{array}\right] .
$$

where $0<\zeta<1$ is damping ratio and $\omega_{n}(t)>0$ is the timevarying bandwidth. The PD-spectral and PD-modal matrices are given by $\Upsilon(t)=\operatorname{diag}\left[\rho_{1}(t), \rho_{2}(t)\right]$ and

$$
M(t)=\left[\begin{array}{cc}
1 & 1 \\
\rho_{1}(t) & \rho_{2}(t)
\end{array}\right]
$$

where $\rho_{1,2}(t)=-\zeta \omega_{n}(t) \pm j \sqrt{1-\zeta^{2}} \omega_{n}(t)$.

$$
\begin{aligned}
P(t) & =\frac{1}{2\left(1-\zeta^{2}\right) \omega_{n}(t)}\left[\begin{array}{cc}
\omega_{n}(t) & \zeta \\
\zeta & \frac{1}{\omega_{n}(t)}
\end{array}\right] \\
Q(t) & =2 \zeta \omega_{n}(t) P(t)
\end{aligned}
$$

with

$$
\begin{gathered}
\lambda(P(t))=\frac{\left(\omega_{n}^{2}(t)+1\right) \pm \sqrt{\left(\omega_{n}^{2}(t)-1\right)^{2}+4 \zeta^{2} \omega_{n}^{2}(t)}}{4 \omega_{n}^{2}(t)\left(1-\zeta^{2}\right)} \\
\kappa(M(t))=\sup _{t \geq t_{0}} \frac{\left(\omega_{n}^{2}(t)+1\right)}{2 \omega_{n}(t) \sqrt{1-\zeta^{2}}} \\
\lambda(Q(t))=2 \zeta \omega_{n}(t) \lambda(P(t))
\end{gathered}
$$

and the exponent of the norm-bound on the trajectory envelope is given by

$$
\mu=\frac{c_{3}}{2 c_{2}}=\inf _{t \geq t_{0}} \frac{\zeta \omega_{n}(t)}{\kappa^{2}(M(t))}
$$

Figures 1-3 illustrate a numerical example with $\zeta=0.707$ and $\omega_{n}(t)$ as shown in Figure 1. Figure 2 and 3 show the elements of $P(t)$ and $Q(t)$. Figure 4 contains a plot of $\kappa\left(\omega_{n}\right)$ and a plot of $\mu\left(\omega_{n}\right)$. *

\section{CONCLUSION AND FUTURE WORK}

In this paper a method has been developed for constructing a quadratic Lyapunov function for an exponentially stable LTV system using a PD-eigenstructure for the system. The result establishes a relationship between Lyapunov first and second methods, providing explicit upper and lower bounds on the Lyapunov function using the singular values of the inverse PD-modal matrix, and an upper bound on the timederivative of the Lyapunov function using the (negative) real-part of the PD-eigenvalue having the smallest magnitude (the slowest mode). An estimate of the exponential envelope of state trajectory is given in terms of condition number of the PD-modal matrix, which determines the peaking, and using the real-part of slowest PD-eigenvalue and the largest singular value of the PDmodal matrix, which determines the exponential decay rate. Illustrative examples are given to show the utility of the result, and to demonstrate fallacy of the frozen-time eigenvalue based stability analysis for LTV systems.

The main result of this paper can be used in further development of the differential algebraic spectral theory (DAST) and DAST-based control techniques such as the trajectory linearization control. It can also be used to extend many known Lyapunov control techniques for timeinvariant systems to time-varying systems. Moreover, it can be used to develop real-time implementable theoretic stability metrics for adaptive control of LTV and linearizable nonlinear time-varying systems.
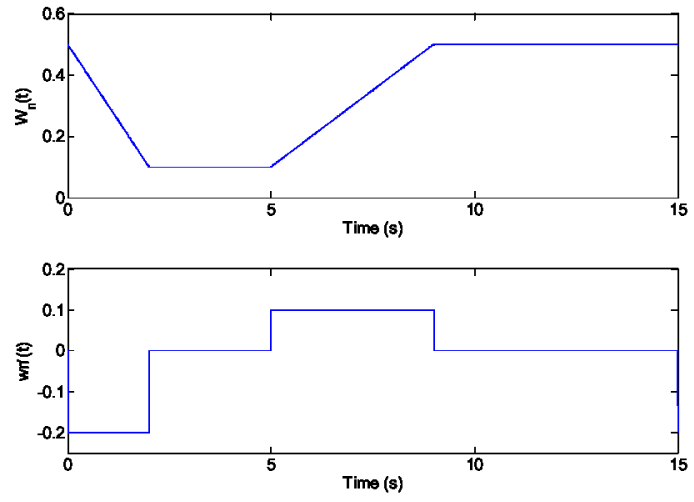

Fig. $1 \omega_{n}(t)$ and $\dot{\omega}_{n}(t)$ 

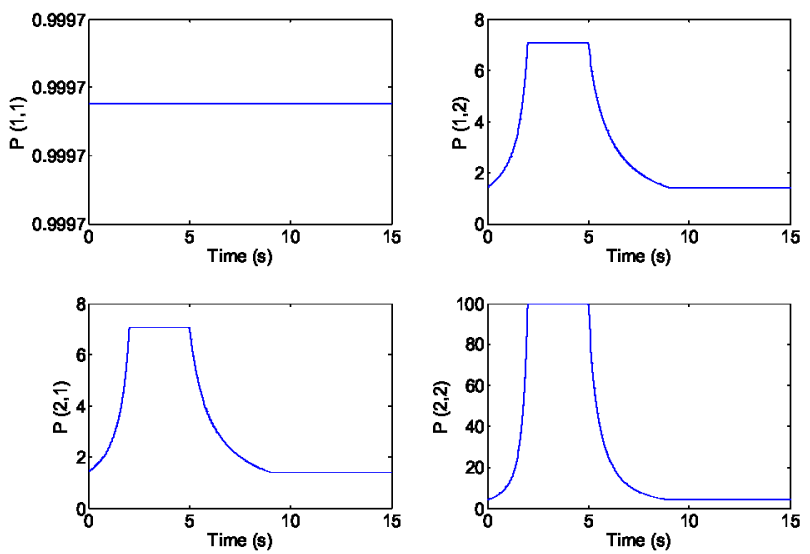

Fig. $2 P(t)$ Trend
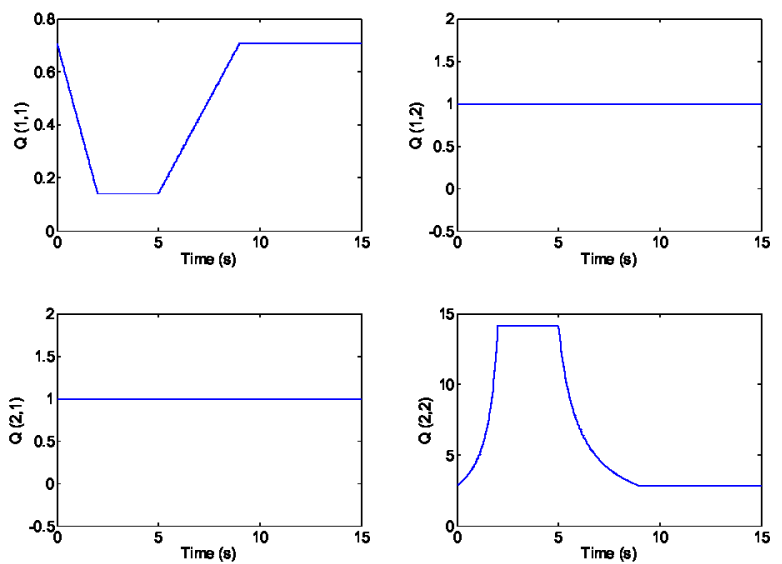

Fig. $3 Q(t)$ Trend
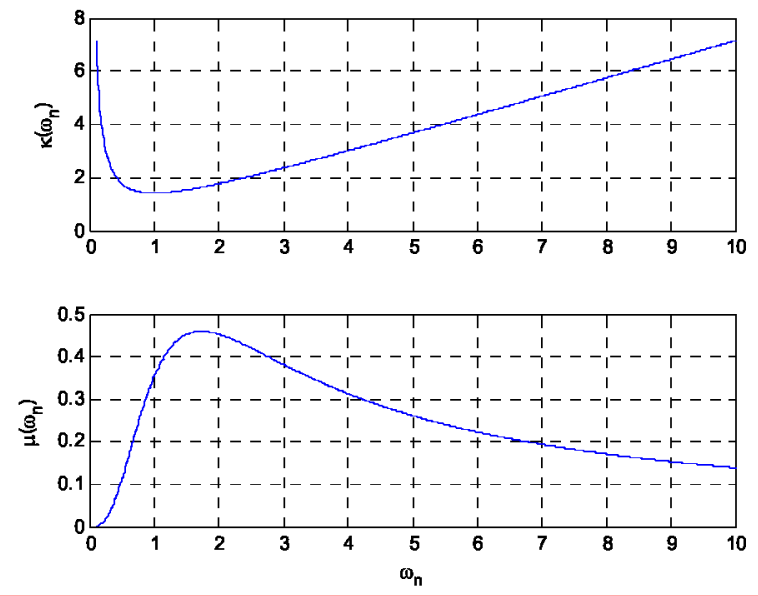

Fig. $4 \kappa\left(\omega_{n}\right)$ and $\mu\left(\omega_{n}\right)$

\section{REFERENCE}

[1] C. L. Pirie, S. Okubo, G. E. Dullerud and D. A. Tortorell, “ Robust linear time-varying control for trajectory tracking: computation and an example", International Journal of Control, vol. 79, no. 4, pp. 349361, April, 2006

[2] C. Pirie and G. E. Dullerud, "Robust controller synthesis for uncertain time-varying systems", SIAM Journal of Control and Optimization, vol. 40, no. 4, pp. 1312-1331, 2002.
[3] W. J. Rugh, and J. S. Shamma, "Research on gain scheduling," Automatica, vol. 36, no. 10, pp. 1401-1425, 2000.

[4] J. Zhu and C. D. Johnson, "Unified canonical forms for matrices over a differential ring," Linear Algebra and Its Applications, vol. 147, 201-248, March 1991.

[5] J. J. Zhu, "A unified spectral theory for linear time-varying systemsprogress and challenges," Proceedings, 34th IEEE Conference on Decision and Control, New Orleans, LA, 2540-2546, Dec. 1995.

[6] J. Zhu, "PD-spectral theory for multivariable linear time-varying systems," Proceedings, 36th IEEE Conference on Decision and Control, San Diego, CA, 3908-3913, Dec. 1997.

[7] J. Zhu, A. S. Hodel, K. Funston and C. E. Hall, "X-33 entry flight controller design by trajectory linearization - a singular perturbational approach," Guidance and Control 2001, Advances in the Astronautical Sciences, Vol. 107, 151-170.

[8] J. J. Zhu, B. D. Banker, and C. E. Hall, "X-33 Ascent Flight Controller Design By Trajectory Linearization-A Singular Perturbational Approach", in Proc. AIAA Guidance, Navigation and Control Conference, Denver, CO, 2000, AIAA-2000-4159.

[9] R. Huang, Y. Liu, and J. Zhu, "Guidance, Navigation and Control System Design for a Tri-Propeller Vertical-Take-Off-and-Landing Unmanned-Air-Vehicle", to appear in Journal of Aircraft.

[10] Y. Liu, R. Williams II, J. J. Zhu and J. Wu, "Omni-Directional Mobile Robot Controller Based on Trajectory Linearization", Robotics and Autonomous Systems, vol. 56, no. 5, 461-479, DOI : 10.1016/j.robot.2007.08.007

[11] J. J. Zhu, D. A. Lawrence, J. Fisher, Y. B. Shtessel, A. S. Hodel and P. Liu, "Direct Fault Tolerant RLV Attitude Control - A Singular Perturbation Approach," in Proc. AIAA Guidance, Navigation and Control, 2002, AIAA2002-4778.

[12] Tony M. Adami, Ryan Sabala and J. Jim Zhu, "Time-varying notch filters for control of flexible structures and vehicles," Proc., 22nd Digital Avionics Systems Conf., Indianapolis, Indiana, Oct. 2003.

[13] H. K. Khalil, Nonlinear Systems, 3rd Ed. Prentice-hall, NJ, 2002.

[14] W. J. Rugh, Linear System Theory, 2nd edition, Prentice Hall, 1995.

[15] K. S. Narendra, and A. M. Annaswanmy, Stable adaptive systems, Prentice Hall, 1989.

[16] A. S. Morse, "Global stability of parameter-adaptive control systems", IEEE Trans. Automatic. Ctrl, vol. 25, no. 3, pp. 433-439, June, 1980.

[17] M. Kristic, I. Kanellakopoulos and P. Kokotovic, Nonlinear and Adaptive Control Design, John Wiley \& Sons, INC, 1995

[18] S. S. Sastry and A. Isidori, "Adaptive control of linearizable systems", IEEE Trans. Auto. Ctrl, vol. 34, no. 11, pp. 1123-1131, Nov. 1989.

[19] R. M. Sannner and J. J. E. Slotine, "Gaussian networks for direct adaptive control", IEEE Trans. Neural Networks, vol. 3, no. 6, pp. 837-863, Nov. 1992.

[20] Z. Gajic and M. Qureshi, Lyapunov matrix equation in system Stability and Control, Academic Press, Inc., San Diego, CA, 1995.

[21] Y-L Zhu and P. R. Pagilla, "Bounds on the solution of the timevarying linear matrix differential equation, " $\dot{P}(t)=A^{H}(t) P(t)+$ $P(t) A(t)+Q(t)$ ", IMA Journal of Mathematical Control and Information, vol. 23, no. 3, pp. 269-277, Sep. 2006.

[22] Y. Liu and J. J. Zhu, "Regular Perturbation Analysis for Trajectory Linearization Control", Proceedings of the 2007 American Control Conference, New York, NY, July 2007, 3053-3058.

[23] Y. Liu, R. Huang and J. J. Zhu, "Adaptive neural network control based on trajectory linearization control", in Proc. 6th World Congress of Intelligent Control Association, Dalian, June, 2006, pp. 417-421.

[24] J. J. Zhu "A note on extension of the eigenvalue concept", Control Systems Magazine, vol. 13, no. 6, pp. 68-70, Dec. 1993.

[25] Wu, M-Y. "On Stability of Linear Time-Varying Systems," Int. J. Systems Sci., Vol. 15, No.2, 137-150, 1984.

[26] Tony Adami and J. Jim Zhu, "Control of a Flexible, Hypersonic Scramjet Vehicle Using a Differential Algebraic Approach," AIAA2008-7464, Proceedings, AIAA Guidance, Navigation and Control Conference, Honolulu, HI, August, 2008.

[27] Tony Adami, J. Zhu, Abraham K. Ishihara, Yoo-Hsiu Yeh, Corey A Ippolito, "Six Degree-of-Freedom Payload-Directed Flight Control by Trajectory Linearization," Proceedings, AIAA InfoTech Conference, Seattle, WA, April, 2009. 